\title{
Oral Health Literacy and Behaviour of Health Sciences University Students
}

\author{
Ng Yee Ying ${ }^{1}$, Lye Shen Ming ${ }^{1}$, Shahida Mohd-Said ${ }^{2}$, Noriah Yusof ${ }^{1}$, Tuti N Mohd-Dom ${ }^{1}$ \\ Department of Dental Public Health, Faculty of Dentistry, Universiti Kebangsaan Malaysia, Kuala Lumpur 50300, \\ Malaysia \\ ${ }^{2}$ Department of Periodontology, Faculty of Dentistry, Universiti Kebangsaan Malaysia, Kuala Lumpur 50300, \\ Malaysia: \\ Correspondence e-mail to: tutinin@ukm.edu.my
}

\begin{abstract}
Objective: This study aimed to determine the level of oral health literacy and behavior among health sciences university students. Methods: The method used descriptive cross-sectional survey involving 609 students from Faculty of Medicine, Dentistry, Pharmacy and Allied Health Sciences in the Universiti Kebangsaan Malaysia. Oral health literacy level and behaviour was assessed with a validated and pretested self-administered questionnaire using the Newest Vital Sign (NVS) tool and modified Oral Health Adult Literacy Questionnaire (OHL-AQ). Results: A total of 509 participants involved in the study (83.6\%). The overall mean oral health literacy score was $10.27(95 \%$ CI 7.92, 12.62), which found dental students showing statistically significant higher scores (mean $=11.36,95 \% \mathrm{CI}$ 9.70, 13.02) compared to medical (mean $=10.72,95 \%$ CI 8.67, 12.77), allied health sciences (mean $=9.89$, 95\% CI $7.34,12.44)$ and pharmacy (mean $=9.55,95 \%$ CI 7.23, 11.87). Almost all respondents are non-smokers $(99.8 \%)$ and non-drinkers (97.2\%). Only 19.1\% pay regular dental visits every 6-12months while $51.1 \%$ visit dentist only when they have dental pain. There appears to be a positive relationship between oral health literacy and oral health behavior. Conclusion: Health science university students should be provided substantial dental health education in their curriculum as they could be potential strategic partners in oral health.
\end{abstract}

Key words: oral health behavior, oral health literacy

\section{INTRODUCTION}

Oral health is defined as a state of being free from chronic mouth and facial pain, oral and throat cancer, oral sores, birth defects such as cleft lip and palate, periodontal disease, tooth decay and tooth loss, and other diseases and disorders that affect the oral cavity. ${ }^{1}$ It also affects the general health, well-being, education and development of children and their families, and diminishes the quality of life. ${ }^{2,3}$

Oral health literacy on the other hand, is defined as "the degree to which individuals have the capacity to obtain, process, and understand basic oral health information and services needed to make appropriate health decisions". ${ }^{4}$ Hence, an individual's health literacy is considered the single best predictor of their health status ${ }^{5}$. Studies have shown that an individual with limited health literacy skills make greater use of services designed to treat complication of disease and less use of services designed to prevent complications. Given the complexity of the healthcare system, it is not surprising that limited health literacy is associated with poor health. Moreover, low health literacy may also have negative psychological effect; those with limited health literacy skills reported a sense of shame about their skill level. As a result, they may hide reading or vocabulary difficulties to maintain their dignity. ${ }^{6}$ Therefore it is crucial to determine whether the public truly understand and benefit from the current oral health education material in order to achieve the National Oral Health Goals 2020 with improved oral health status among Malaysians. ${ }^{?}$

The Ministry of Health recognizes that improving the health of Malaysians can only be achieved by key organizations working together at all levels since members of the public make more frequent contacts with the primary care sector. ${ }^{7}$ Thus, primary health care teams are ideally placed to promote oral health within the context of general health, reinforcing the possibility that dental health teams can benefit from having allies beyond the oral health community. ${ }^{8}$ Thus it is also equally important to ensure that all members 
of the health profession have high level of oral health literacy as they have the potential to promote oral health by supporting and spreading accurate oral health messages, showing exemplary oral health-related behavior, and encouraging appropriate dental visits.

Very few studies have been done to assess the oral health literacy of undergraduates in local universities. This study will determine the oral health literacy and behavior of the first year undergraduate students who have at least one year of basic science foundation and would later be collaborative partners in health. By virtue of the fact that they have at least one year of basic science foundation, they should have certain level of oral health literacy compared to the young adults who have not been exposed to the science field. This could also be a baseline study to help to further expand and develop appropriate oral health education material to further improve oral health awareness and practice. This study aims to determine the level of oral health literacy and behavior among medical, dental, pharmacy and allied health sciences first year undergraduate students of Universiti Kebangsaan Malaysia (UKM). The objectives of this study are to determine and correlate (i) the social demographic profile, (ii) the level of oral health literacy and (iii) the oral health behavior of the cohort.

\section{METHODS}

\section{Study Sample}

This descriptive cross-sectional survey was conducted using self-administered questionnaires. All first year undergraduates in the Faculty of Medicine, Dentistry, Pharmacy, and Allied Health Sciences in the UKM who have at least one year of basic science foundation were invited to participate in this study. Those who agreed to take part in this study were asked to complete a set of questionnaires on age, gender, course of study, oral health literacy level related to reading comprehension, numeracy skills and appropriate decision-making skills; and oral health behavior related to daily oral hygiene care, dietary habit, alcohol and smoking habit, and dental visits. The questionnaire forms were distributed to the students at the end of scheduled class sessions with permission of the respective deans of faculty. The activity took an average of fifteen minutes to complete and the forms were retrieved immediately after the sessions. Students who were absent during the duration of data collection were excluded from the study. Approval to conduct the study including ethics clearance was obtained from UKM Research and Ethics Committee [UKM 1.5.3.5/244/DD/2014/030(2)].

\section{Questionnaires}

The questionnaire was adopted and modified from the Newest Vital Sign (NVS) tool and Oral Health Literacy-Adult Questionnaire (OHL-AQ) ${ }^{4}$ It was designed to determine the oral health literacy level of the students as well as self-reported oral health behavior. Response formats included forced choice format in which respondents choose one or more responses from a provided list of options, write-in the response, or perform a combination of the two. The respondents were made aware that they may have more than one response for some items. Prior to the survey, the questionnaire was pre-tested with thirty students of matching criteria from University of Malaya to assess the clarity and to estimate the time needed to complete the questionnaire.

\section{Oral Health Literacy}

Oral health literacy level was measured by asking fourteen questions related to reading comprehension, numeracy skills and appropriate decision-making skills. The Newest Vital Sign tool was included in this section to measure the level of numeracy skills. An answer was scored incorrect (0 mark) when the chosen or written response was wrong, while an answer was scored correct (1 mark) when the chosen or written response was correct. Total scores ranged from 0 (no correct answer given) to 14 (gave all correct answers). For this study, a score of more than 7 marks suggests adequate oral health literacy.

\section{Oral Health-Related Behavior}

The questions asked were related to daily oral hygiene practice (frequency of tooth brushing, types of toothpaste used), dietary habit (frequency of sugary consumption per day, frequency of snacking in between meals), alcohol and smoking habits, and dental visits (frequency of dental visit, reason for last dental visit). There were a total of eight questions. A score of one point was given for desirable oral health behavior while zero score was given for undesirable oral health behavior. Total scores ranged from 0 (very poor oral health behavior) to 8 (very good oral health behavior).

\section{Statistical Analysis}

Data management and statistical analysis were carried out using Statistical Package for Social Science version 22.0 (SPSS Inc). Oral health literacy and oral health behavior were presented using frequency distributions for each item for each student group. Mean oral health literacy scores and 95\% confidence interval for each student group was calculated and compared using Analysis of variance (ANOVA).

\section{RESULTS}

Six hundred and nine questionnaires were distributed whereby 509 were returned and fully completed with an overall response rate of $83.6 \%$. The highest proportion of respondents in this study was from the Allied Health Sciences (FSK) students at 43.0\% (Table 1). Almost $80 \%$ of the respondents were females while the mean age was 20.3 years old (S.D 0.7 years). 
Table 1. Response rate of study participants $(n=509)$

\begin{tabular}{lccccc}
\hline \multirow{2}{*}{ Course of study } & \multicolumn{4}{c}{ Characteristics } & \\
\cline { 2 - 5 } & \multicolumn{2}{c}{ Total enrolment } & Number participated & Response rate \\
Medicine & Frequency (n) & Percentage (\%) & Frequency (n) & Percentage (\%) & $(\%)$ \\
Dentistry & 195 & 32.0 & 150 & 29.5 & 76.9 \\
Pharmacy & 67 & 11.0 & 64 & 12.6 & 95.5 \\
FSK & 92 & 15.1 & 76 & 14.9 & 82.6 \\
\hline TOTAL & 255 & 41.9 & 219 & 43.0 & 85.9 \\
\hline
\end{tabular}

Table 2. Oral health literacy for each category of assessment

\begin{tabular}{lccccc}
\hline \multirow{4}{*}{ Oral health literacy } & \multicolumn{2}{c}{ Number of respondents answered correctly (\%) } & \multirow{2}{*}{ Total (\%) } \\
& Medical & Dental & Pharmacy & FSK & \\
& $n=150$ & $n=64$ & $n=76$ & $n=219$ & $n=509$ \\
Reading comprehension & $100(66.6)$ & $46(71.9)$ & $52(68.4)$ & $145(66.2)$ & $343(67.3)$ \\
Numeracy & $134(89.3)$ & $56(87.5)$ & $59(77.6)$ & $182(83.1)$ & $431(84.3)$ \\
Appropriate decision-making & $105(70.0)$ & $55(85.9)$ & $44(57.9)$ & $121(55.3)$ & $325(63.8)$ \\
\hline
\end{tabular}

It was found that on average $71.8 \%$ have adequate level of oral health literacy (good reading comprehension skills $=67.3 \%$, numeracy $=84.3 \%$ and decision-making $=63.8 \%)($ Table 2$)$. The overall mean oral health literacy score was 10.27 (95\% CI 7.92, 12.62), which found the dental students showing statistically significant (ANOVA, $\mathrm{p}<0.01$ ) higher mean scores at $11.36 \pm 1.66$ $($ mean + SD) $(95 \%$ CI 9.70, 13.02) compared to medical $(10.72+2.05,95 \%$ CI 8.67, 12.77), FSK $(9.89 \pm 2.56$, $95 \%$ CI $7.34,12.44)$ and pharmacy $(9.55 \pm 2.32,95 \%$ CI $7.23,11.87$ ).

For reading comprehension skills, majority of respondents answered correctly to the question on forms of sugar $(80.0 \%)$ but only about half of the respondents answered correctly to the question on snacking behavior $(51.5 \%)$ (Table 3$)$. For numeracy skills, majority of respondents answered correctly to the questions on drug prescription $(87.2 \%)$, usage of mouthwash $(91.7 \%)$ and instructions after using mouthwash $(95.1 \%)$. However based on the scores on nutrition facts from the NVS tool, only $66.6 \%$ respondents showed adequate literacy, $27.3 \%$ have possibility of limited literacy and $9.4 \%$ showed high likelihood of limited literacy. On the other hand for decision-making skills, more than half of the respondents answered correctly to the questions on how to remove calculus $(60.7 \%)$ and allergic reaction to drugs $(81.7 \%)$ but less than half of the respondents were able to select the proper management when bleeding after brushing teeth occurs (49.1\%).
With regards to oral health behavior (Table 4), a majority of $93.9 \%$ reported that they brush their teeth at least twice daily. However, only $16.5 \%$ reported the usage of dental floss. Generally, $73.9 \%$ consumed sugary snacks and carbonated drinks 1-2 times daily, while $38.7 \%$ consumed sweetened snacks or carbonated drinks in between meals (Table 5). Almost all respondents are non-smokers $(99.8 \%)$ and non-drinkers (97.2\%). Only $19.1 \%$ pay regular dental visits every 6-12 months while half (51.1\%) visit dentist only when they have dental pain (Table 6).

\section{DISCUSSION}

Oral health is an integral part of general health thus oral healthcare was given considerable importance in Malaysia during the last decade. However, very few studies have been done to assess the oral health literacy of undergraduates who have had at least one year of basic science foundation. As future healthcare providers as well as the representative of the young adult population, the result could be a baseline to help to further expand and develop appropriate oral health education material to further improve oral health awareness and practice for young adults in the public. Literature also suggests that higher oral health literacy was found to be associated with better oral health status ${ }^{9}$ and the lack of dental knowledge and poor oral health behavior could be overcome by incorporating oral health module in undergraduate training programs. ${ }^{10}$ 
Table 3. Oral health literacy $(n=509)$

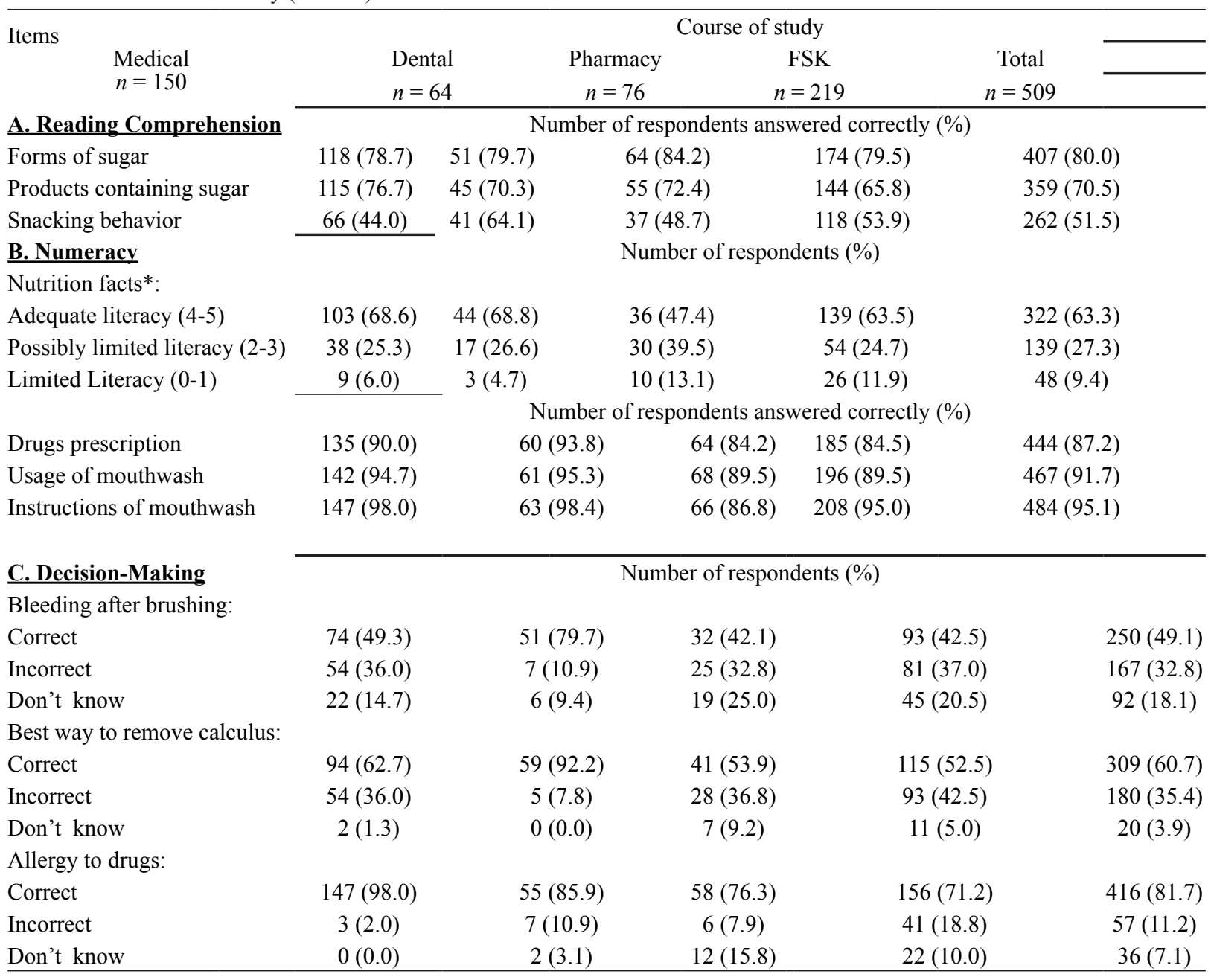

*Nutrition facts: 1 mark is given for each correct answer (maximum 5 marks); Score of 4-5 indicates adequate literacy; Score of 2-3 indicates the possibility of limited literacy; Score of 0-1 suggests high likelihood of limited literacy

In general, this study found that the juniors in medical and health courses have adequate oral health literacy and good oral health behavior. Among the three categories of assessment on oral health literacy i.e. reading comprehension, numeracy, decision-making skills, appropriate decision-making skills need to be further improved among the students. Majority of the non-dental students answered wrongly to the questions on management of bleeding gums and the best method to remove calculus. This shows that their oral health knowledge is not at par with their dental colleagues, most likely owing to the lack of oral health education in their curriculum.

The results indicate that students have good daily oral health practice as majority of them brush their teeth at least twice daily before breakfast and before night sleep using fluoridated toothpaste. However, on average, only $16.5 \%$ reported the use of floss, which is comparable to a recent study in Malaysia where only $8.4 \%$ of Malaysian population reported the use of floss. ${ }^{11}$ Interestingly, almost half of the dental students claimed to use floss once daily despite they had just started the course of study. Dental students reported to have had more regular dental check-ups compared to others. This may be because oral hygiene practice was already taught in the curriculum, or the respondents may be over reporting. Due consideration was also made for the fact that self-administered surveys may not always reflect the true behaviour of the respondents as bias may occur when the respondents do not provide an honest response resulting in under or over reporting of the actual behaviour. The respondent may have believed the information they report (self-deception), 
Table 4. Daily oral health practice of respondents $(n=509)$

\begin{tabular}{|c|c|c|c|c|c|}
\hline \multirow{3}{*}{ Daily Oral Health Practice } & \multicolumn{5}{|c|}{ Number of respondents (\%) } \\
\hline & Medical & Dental & Pharmacy & FSK & Total \\
\hline & $\mathrm{n}=150$ & $\mathrm{n}=64$ & $\mathrm{n}=76$ & $\mathrm{n}=219$ & $\mathrm{n}=509$ \\
\hline \multicolumn{6}{|l|}{ Toothbrushing frequency: } \\
\hline Once or less daily & $11(7.3)$ & $3(4.7)$ & $4(5.3)$ & $13(5.9)$ & $31(6.1)$ \\
\hline Twice or more daily & $139(92.7)$ & $61(95.3)$ & $72(94.7)$ & $206(94.1)$ & $478(93.9)$ \\
\hline \multicolumn{6}{|l|}{ Usual brushing time: } \\
\hline Morning before breakfast & $134(89.3)$ & $57(89.1)$ & $75(98.7)$ & $212(96.8)$ & 478 (93.9) \\
\hline Morning after breakfast & $12(8.0)$ & $4(6.3)$ & $1(1.3)$ & $7(3.2)$ & $24(4.7)$ \\
\hline Before night sleep & $123(82.0)$ & $57(89.1)$ & $69(90.8)$ & $195(89.0)$ & $444(87.2)$ \\
\hline Lunch & $21(14.0)$ & $9(14.1)$ & $11(14.5)$ & $35(16.0)$ & $76(14.9)$ \\
\hline After every meal & $17(11.3)$ & $4(6.3)$ & $2(2.6)$ & $20(9.1)$ & $43(8.4)$ \\
\hline \multicolumn{6}{|l|}{ Cleaning in between teeth: } \\
\hline Toothbrush + toothpaste & $106(70.7)$ & $24(37.5)$ & $54(71.1)$ & $154(70.3)$ & $338(66.4)$ \\
\hline Mouthwash & $16(10.7)$ & $8(12.5)$ & $11(14.5)$ & $26(11.9)$ & $61(12.0)$ \\
\hline Dental Floss & $20(13.3)$ & $31(48.4)$ & $8(10.5)$ & $25(11.4)$ & $84(16.5)$ \\
\hline Toothpick & $8(5.3)$ & $1(1.6)$ & $3(3.9)$ & $13(5.9)$ & $25(4.9)$ \\
\hline Others & $0(0.0)$ & $0(0.0)$ & $0(0.0)$ & $1(0.5)$ & $1(0.2)$ \\
\hline \multicolumn{6}{|l|}{ Type of toothpaste: } \\
\hline Fluoridated & $127(84.7)$ & $60(93.8)$ & $63(82.9)$ & $168(76.7)$ & $418(82.1)$ \\
\hline Non-fluoridated & $11(7.3)$ & $2(3.1)$ & $7(9.2)$ & $28(12.8)$ & $48(9.4)$ \\
\hline Herbal & $11(7.3)$ & $2(3.1)$ & $6(7.9)$ & $22(10.0)$ & $41(8.1)$ \\
\hline Others & $1(0.7)$ & $0(0.0)$ & $0(0.0)$ & $1(0.5)$ & $2(0.4)$ \\
\hline \multicolumn{6}{|l|}{ Change of toothbrush: } \\
\hline Every 3 months & $91(60.7)$ & $45(70.3)$ & $38(50.0)$ & $117(53.4)$ & $291(57.2)$ \\
\hline Every 6 months & $30(20.0)$ & $14(21.9)$ & $14(18.4)$ & $45(20.5)$ & $103(20.2)$ \\
\hline Every year & $3(2.0)$ & $0(0.0)$ & $2(2.6)$ & $10(4.6)$ & $15(2.9)$ \\
\hline More than 1 year & $1(0.7)$ & $0(0.0)$ & $0(0.0)$ & $0(0.0)$ & $1(0.2)$ \\
\hline When it has split ends & $25(16.7)$ & $5(7.8)$ & $22(28.9)$ & $47(21.5)$ & $99(19.4)$ \\
\hline
\end{tabular}

or may 'fake good' to conform to socially acceptable values, avoid criticism, or gain social approval. ${ }^{12,13}$

Findings regarding dietary habit indicate that slightly more than half of the students did not consume sugary snack in between meal. This is fairly acceptable but awareness and knowledge could be increased further so that more students change their dietary habit and stop sugary snacking in between meals totally. Studies have shown that frequency of sugary consumption is a more crucial factor in caries formation compared to its amount. The Vipeholm study established that sticky sweets consumed between meals (rather than within meals) significantly increased carious tooth surface formation. ${ }^{14}$
Interestingly, there appears to be positive relationship between oral health literacy and oral health behavior. This is especially evident via the questions regarding snacking behavior. About $40 \%$ of the respondents reported to snack in between meals, which could explain why half of the respondents answered wrongly to the question on which snacking behavior is more detrimental to oral health. This finding is consistent with the findings of other studies showing that limited health literacy is associated with fewer healthy lifestyle behaviors. Based on a study conducted by Masayuki Ueno et al, the higher a participant's oral health literacy, the more often they brushed their teeth or dentures, self-checked oral condition with a mirror, had regular dental checkups, and the better their oral hygiene status. $^{15}$ 
Table 5. Dietary habit of respondents $(n=509)$

\begin{tabular}{|c|c|c|c|c|c|}
\hline \multirow{3}{*}{ Dietary Habit } & \multicolumn{5}{|c|}{ Number of respondents $(\%)$} \\
\hline & Medical & Dental & Pharmacy & FSK & Total \\
\hline & $\mathrm{n}=150$ & $\mathrm{n}=64$ & $\mathrm{n}=76$ & $\mathrm{n}=219$ & $\mathrm{n}=509$ \\
\hline \multicolumn{6}{|c|}{$\begin{array}{l}\text { Frequency of sugary con- } \\
\text { sumption: }\end{array}$} \\
\hline 1-2 times & $101(67.3)$ & $44(68.8)$ & $59(77.6)$ & $172(78.5)$ & $376(73.9)$ \\
\hline 3-4 times & $18(12.0)$ & $9(14.1)$ & $7(9.2)$ & $11(5.0)$ & $45(8.8)$ \\
\hline$>4$ times & $7(4.7)$ & $1(1.6)$ & $2(2.6)$ & $3(1.4)$ & $13(2.6)$ \\
\hline Never & $24(16.0)$ & $10(15.6)$ & $8(10.5)$ & $33(15.1)$ & $75(14.7)$ \\
\hline \multicolumn{6}{|c|}{ Snacking between meals: } \\
\hline Yes & $65(43.3)$ & $26(40.6)$ & $36(47.4)$ & $70(32.0)$ & $197(38.7)$ \\
\hline \multirow[t]{2}{*}{ No } & $85(56.7)$ & $38(59.4)$ & $40(52.6)$ & $149(68.0)$ & $312(61.3)$ \\
\hline & \multicolumn{5}{|c|}{ Number of respondents $(\%)$} \\
\hline \multirow[t]{2}{*}{ Dental Visits } & Medical & Dental & Pharmacy & FSK & Total \\
\hline & $\mathrm{n}=150$ & $\mathrm{n}=64$ & $\mathrm{n}=76$ & $\mathrm{n}=219$ & $\mathrm{n}=509$ \\
\hline \multicolumn{6}{|l|}{ Frequency: } \\
\hline Regular & $20(13.3)$ & $25(39.1)$ & $6(7.9)$ & $46(21.0)$ & $97(19.1)$ \\
\hline Symptomatic & $78(52.0)$ & $19(29.7)$ & $45(59.2)$ & $118(53.9)$ & $260(51.1)$ \\
\hline Occasionally & $35(23.3)$ & $17(26.6)$ & $21(27.6)$ & $40(18.3)$ & $113(22.2)$ \\
\hline Never & $17(11.3)$ & $3(4.7)$ & $4(5.3)$ & $15(6.8)$ & $39(7.7)$ \\
\hline \multicolumn{6}{|l|}{ Last visit: } \\
\hline$<2$ years ago & $96(64.0)$ & $60(93.7)$ & 49 (64.4) & $161(73.5)$ & $366(71.9)$ \\
\hline$>2$ years ago & $54(36.0)$ & $4(6.3)$ & $27(35.6)$ & $58(26.5)$ & $143(28.1)$ \\
\hline \multicolumn{6}{|c|}{ Reason for visit: } \\
\hline Preventive & $81(54.0)$ & $29(45.3)$ & 44 (57.9) & $142(64.8)$ & $296(58.2)$ \\
\hline Symptomatic & $69(46.0)$ & $35(54.7)$ & $32(42.1)$ & 77 (35.2) & $213(41.8)$ \\
\hline
\end{tabular}

Fortunately, although majority of the respondents have close relatives who smoke and drink alcohol, more than $95 \%$ of them were non-smokers and non-drinkers, showing that they were not influenced by external factors. This reflects that the first year undergraduate students have commendable oral health behaviour, in view of their future profession as key players in promoting and providing general and oral healthcare to the public.

\section{CONCLUSION}

There appears to be a positive relationship between oral health literacy and oral health behavior. The majority has adequate oral health literacy and good oral health behavior, showing good potential as future collaborative partners in health and oral health promotion.

\section{ACKNOWLEDGMENT}

This study was funded by the UKM Strategic/Action Plan Research Grant (PTS 2014-054). The authors are indebted to Associate Professor Dr Aznida Firzah Abdul Aziz and Deans of Faculty of Medicine, Dentistry, Pharmacy and Allied Health Sciences for their contribution and support. 


\section{REFERENCES}

1. World Health Organization [Internet]. Oral Health. Fact sheet No 318. [cited April 2012. Available from: http://www.who.int/mediacentre/factsheets/ fs318/en/.

2. Kwan SY, Petersen PE, Pine CM, Borutta A. Health-promoting schools: an opportunity for oral health promotion. Bulletin of the World Health Organization. 2005. 83: 677-685.

3. Lateefat S, Musa OI, Kamaldeen AS, Muhammad AS, Saka OI. Determinants of Oral Hygiene Status among Junior Secondary School Students in Ilorin West Local Government Area of Nigeria. IOSR J Pharm Biol Sci 2012;1:44-48.

4. Naghibi SM, Montazeri A, Yazdani R. New oral health literacy instrument for public health: development and pilot testing. J Invest Clin Dent. 2013;5(4):313-321.

5. Badarudeen S, Sabharwal S. Assessing Readability of Patient Education Materials: Current Role in Orthopaedics. Clin Orthop Relat Res. 2010;468(10): 2572-2580.

6. U.S Department of Health and Human Services, Office of Disease and Health Promotion. Quick Guide to Health Literacy, Health Literacy Basics. 2010.

7. Oral Health Division, Ministry of Health Malaysia. National Oral Health Plan for Malaysia 2011-2020. 2011.
8. Tuti NM, Shahida MS, Zamirah ZA. Dental Knowledge and Self-reported Oral Care Practices among Medical, Pharmacy and Nursing Students. Mal J Heatlh Sci. 2009;7:13-23.

9. Lee JY, Divaris K., Baker AD, Rozier RG, Vann, WF. The Relationship of Oral Health Literacy and Self-Efficacy with Oral Health Status and Dental Neglect. Am J Public Health. 2012;102:923-9.

10. Graham E, Negron R, Domoto P, Milgrom P. Children's oral health in the medical curriculum: a collaborative intervention at a university-affiliated hospital. J Dent Educ. 2003;67:338-47.

11. Umer A,. Oral health care in Malaysia - A review. Pak Oral Dent J. 2011;31. King, M, Bruner G. Social desirability bias: a neglected aspect of validity testing. Psychol Market. 2000;17:79-103.

12. Huang C, Liao H, Chang S. Social desirability and the Clinical Self-Report Inventory: methodological reconsideration. J Clin Psychol. 1998;54:517-28.

13. Krasse B. The Vipeholm Dental Caries Study: Recollections and Reflections 50 Years Later. J Dent Res. 2001.80:1785-8.

14. Ueno M, Takeuchi S, Oshiro A, Kawaguchi Y. Relationship between oral health literacy and oral health behaviors and clinical status in Japanese adults. JDS. 2012;8: 170-6. Available from: http://www.e-jds.com/article/S19917902\%2812\%2900154-7/fulltext.

(Received July 1, 2015; Accepted August 28, 2015) 